1 RUNNING HEAD: PHYSICAL EXERCISE AFFECTS DECISION MAKING

2

3 Attentional and perceptual capabilities are affected by high physical load in a simulated soccer decision-making task 
Abstract

7 In a sport-specific decision-making task, we investigated whether different intensities of physical

8 load have different effects on soccer players' decision making, visual attention, and perception.

9 Under a rest condition as well as under physical exercise conditions of 70\% (Moderate Load)

10 and $90 \%$ (High Load) of their heart rate reserve, participants $(N=30)$ performed a soccer-related

11 decision-making, a feature-recognition and an object-detection task in front of an immersive

12 screen. Stimuli were displayed across a range of 0 to 180 degree visual angles. Results showed

13 that decision-making performance decreased with increasing visual angles but was not

14 negatively affected by physical demands. However, perceptual and attentional capabilities

15 remained constant in the Moderate Load condition and deteriorated in the High Load condition

16 compared to the rest condition. Furthermore, in the High Load condition, perceptual capabilities

17 decreased more drastically with increasing visual angles compared to the other conditions. The

18 findings show that high physical load affects attentional and perceptual capabilities more than

19 moderate physical load, while decision-making performance does not differ in both conditions.

21 Keywords: object-detection; feature-recognition; physical load; sport expertise; training 


\section{Attentional and perceptual capabilities are affected by high physical load in a simulated} soccer decision-making task

In order to be successful, athletes must rapidly perceive the positions and movements of teammates and opponents as well as the ball, attend to the most relevant features of the match, and make correct decisions (Hüttermann, Ford, Williams, Varga, \& Smeeton, 2019; Klatt \& Smeeton, 2020). In different sport situations, especially in most team sports, athletes are usually required to rapidly make these complex decisions under time pressure and high physical demands (Zurutuza, Castellano, Echeazarra, \& Casamichana, 2017). In order to investigate this

31 area of research, some studies have examined the effects of physical exercise on sport athletes'

32 overall decision-making performance (e.g., Hepler, 2015). Others have investigated components

33 of decision making such as the link between physical exercise and attentional performance in

34 athletes (e.g., Pesce, Cereatti, Casella, Baldari, \& Capranica, 2007) and the relationship between

35 physical exercise and perceptual performance (e.g., Swart, Lindsay, Lambert, Brown, \& Noakes,

36 2012). To date, however, there has been no examination of the effect of physical exercise on

37 attentional and perceptual tasks underpinning decision making in sport $-\mathrm{a}$ decision that needs to

38 be made rapidly in a complex environment. The aim of the current study, therefore, was to

39 examine the effect of physical exercise on both athletes' attentional and perceptual capabilities in 40 a single sport-specific decision-making task.

41 Over the last decades, several classes of theoretical models have been proposed to explain

42 the effects of exercise on cognitive performance. One of the most dominant models suggests an

43 inverted U-shape relationship to describe the change in cognitive performance as exercise 44 intensity increases (cf. Yerkes-Dodson Law; Yerkes \& Dodson, 1908). A moderate level of 45 exercise usually results in increased physiological arousal and facilitated cognition compared to a 46 rest condition. Furthermore, as soon as physiological arousal approaches a maximum level, 
47 cognitive performance often declines (e.g., Chmura, Nazar, \& Kaciuba-Uscilko, 1994; McMorris

48 \& Graydon, 2000; McMorris, Sproule, Turner, \& Hale, 2011). Alternatively, arousal-based

49 models suggest that exercise increases arousal and when the physical load is too high, the

50 demand for resources is beyond the amount available and performance declines as a result

51 (Humphreys \& Revelle, 1984; Lambourne \& Tomporowski, 2010; Sanders, 1983). However, the

52 inconsistent results from studies makes it difficult to rule out any one model.

53 Inconsistent results from studies examining the effect of exercise on cognition have been

54 attributed to the type of task (for a review, see Tomporowski, 2003), exercise intensity (e.g.,

55 Labelle, Bosquet, Mekary, \& Bherer, 2013), duration and mode (e.g., Lambourne \&

56 Tomporowski, 2010) as well as sport-specific effects (e.g., McMorris \& Graydon, 1997) and

57 expertise and fitness effects (e.g., Hüttermann \& Memmert, 2014). Studies that have examined

58 the task performed have shown effects for simple detection tasks (e.g., McMorris and Keen,

59 1994), visual search tasks (e.g., Aks, 1998; Allard, Brawley, Deakin, \& Elliot, 1989),

60 discriminative choice-response tasks (e.g., Arcelin, Brisswalter, \& Delignières, 1997;

61 Delignières, Brisswalter, \& Legros, 1994), and complex problem-solving tasks (e.g., McMorris

62 et al., 1999; Tenenbaum, Yuval, Elbaz, Gar-Eli, \& Weinberg, 1993). For example, Lambourne,

63 Audiffren, and Tomporowski (2010) showed that a sensory detection task performance was

64 facilitated during 40 minutes of exercise at $90 \%$ below ventilatory threshold compared to rest

65 performance. No change was found for a cognitive task during exercise. These results suggest

66 that steady state exercise at an intensity below ventilatory threshold influences sensory but not

67 central executive task function. Whilst these experimental designs offer experimental control,

68 they do not replicate the physical demands (i.e. intermittent exercise) of sports that require

69 complex decisions to be made. 
There are some studies that have investigated physical exercise effects on cognition in sport-specific situations. McMorris and Graydon (1996) investigated the impact of physical

72 exercise on a visual information processing and searching in a decision-making task in soccer.

73 Experienced soccer players exercised at $70 \%$ or $100 \%$ of their maximum power output and

74 made decisions in comparison to rest. They found that maximal exercise facilitated visual search

75 and speed of ball detection was faster during exercise. Additionally, Royal et al. (2006) found

76 that sport-specific tests of decision making during a very high fatigue (high exercise intensity)

77 condition facilitate decision making, but not motor performance, in water polo. Especially in

78 fast-paced team sports, such as water polo and soccer, well-developed visual and attentional

79 skills are required to enable players to make the right decisions under time pressure. In a recently

80 published study by Hüttermann, Smeeton, Ford, and Williams (2019), these visual and

81 attentional skills were examined in one sport-specific test. The authors developed a soccer-

82 specific task to examine decision making as a function of attentional and perceptual capabilities.

83 Stimuli in the form of pairs of soccer players were briefly presented across a range of visual

84 angles on a large immersive screen (radius of $3 \mathrm{~m}$ ). Participants were required to decide to whom

85 to pass the ball to while their perceptual and attentional skills on this task were assessed. Results

86 showed attentional performance was poorer than perceptual performance when stimuli were

87 presented across wider viewing angles (cf. Hüttermann, Ford et al., 2019 for similar results

88 concerning the same soccer-specific task as well as Hüttermann and Memmert, 2017 for a

89 general distinction between attentional and perceptual skills). What is unclear is how perceptual

90 and cognitive processes involved in sport-specific decision making are influenced by high

91 physical loads often experienced when playing sports.

92 The physical load of elite soccer players during games has been well-described (e.g.,

93 Sarmento et al., 2014). Various studies included both acceleration and metabolic variables (e.g., 
94 Dalen, Ingebrigtsen, Ettema, Havard, \& Wisløff, 2016; Osgnach, Poser, Bernardini, Rinaldo, \&

95 Di Prampero, 2010; Russell et al., 2014). Soccer involves intermittent sprinting activity and,

96 whilst there are positional and time-of-game-specific differences, there are periods of high,

97 medium, and low intensity activity separated by active and passive recovery periods (Bradley et

98 al., 2009). The effect of subjecting athletes to physical loads with short duration periods on

99 decision making has not yet been examined in detail. The aim of the current study, therefore, was

100 to explore the effect of short duration periods of moderate and high physical loads on soccer

101 players' performance in the sport-specific decision-making task validated by Hüttermann,

102 Smeeton, and colleagues (2019). In this task, participants are required to judge two stimuli

103 equidistant to the centre of an immersive screen at their left and right body side with varying

104 visual angles between the stimuli. Each stimulus consists of a player configuration of one

105 teammate and a maximum of three opponent players. Participants then have to decide on whether

106 and where to pass the ball (decision-making task), they also have to perceive the movement

107 direction of their teammates (feature-recognition task), and they have to recognize the number of

108 opponent players surrounding their teammates (object-detection task). While the object-detection

109 task requires the differentiation between jersey colours (recognition of number of players

110 wearing white jerseys), the feature-recognition task requires the differentiation between colour

111 and shape of stimuli (recognition of players wearing black jerseys and assessment of their

112 running direction) thereby, demanding more visual attention (cf. Hüttermann, Ford et al., 2019).

113 In order to present the game situations in a realistic size in foveal and peripheral vision a $210^{\circ}$

114 immersive dome with a radius of $3 m$ was used (cf. Klatt \& Smeeton, 2019). Participants

115 performed the decision-making task at rest, at a moderate, and at a high physical load condition.

116 Based on previous findings showing a link between physical exercise, visual (e.g., McMorris \&

117 Graydon, 1997) and attentional performance (e.g., Hüttermann \& Memmert, 2014) as well as 
118 decision making (e.g., Hepler, 2015; Paradis, Larkin, \& O’Connor, 2016), we assumed that

119 athletes' perception (Lambourne et al., 2010), visual attention (Hüttermann \& Memmert, 2014),

120 and decision making (Royal et al., 2006) in the soccer-specific task would be affected by

121 changes in the physical load. More precisely, we expected changes to task performance to be

122 seen between moderate exercise load (70\% of heart rate reserve) and high exercise load (90\% of

123 heart rate reserve), and compared to the rest condition.

\section{Method}

\section{Sample size estimation}

126 Based on previous research examining the attentional window and decision making in

127 sport (Hüttermann, Ford et al., 2019; Hüttermann, Smeeton et al., 2019; Klatt \& Smeeton, 2020),

128 a minimum sample size of 28 was calculated using $\mathrm{G}^{*}$ Power (Faul, Erdfelder, Buchner, \& Lang,

129 2009). This calculation was based on the main effect of visual angle in these previous studies

130 having a median effect size $\left(\eta^{2}\right)$ of .623 and a $50 \%$ attenuation of this variable under different

131 exercise loads being predicted.

\section{Participants}

Altogether, 30 participants ( 6 female) aged 19 to 28 years $\left(M_{\text {age }}=23.97\right.$ years, $S D=2.34$

134 years) took part in the experiment. Data from three additional participants had to be excluded

135 because one had muscular problems restricting the exercise performance during the task, one had 136 circulatory problems, and one did not reach the chance level threshold for performance in any of

137 the tasks. According to self-reports, all other participants were healthy, and physically active.

138 Twenty-six participants were active soccer players and reportedly participated in competitions

139 regularly (e.g., in the English national league, national league south, southern league), the other

140 four also had previous experience in playing soccer for at least ten years. (Experiences in soccer

141 for at least ten years were provided in order to take part at the study.) Overall, participants had 
142 played soccer for 12.00 years $(S D=1.76$ years $)$. At the time of the data collection, they trained

143 for an average of 9.87 hours $(S D=2.08$ hours) on the soccer field per week. Twenty-three

144 players reported to usually prefer kicking with their right leg/foot and seven players with their

145 left leg/foot. Furthermore, participants reported normal or corrected-to-normal (with contact

146 lenses) vision - this was another prerequisite in order to take part. The study was carried out in

147 accordance with the Helsinki Declaration of 1975, and written informed consent was obtained

148 from each participant prior to testing. Approval was obtained from the lead institution's ethics

149 board.

$150 \quad$ Materials and Procedure

151 Participants were tested individually in a laboratory room. They sat on a cycle ergometer

152 (Wattbike Pro Indoor Trainer ${ }^{\circledR}$ ) at a distance of $3 \mathrm{~m}$ from the centre of a $210^{\circ}$ curved projection

153 screen (IGLOO, radius of $3 \mathrm{~m}$, height: 2.20m; see Figure 1). They wore a heart rate monitor

154 (Polar A300®), and their heart rate as well as cadence were continuously monitored during the

155 whole testing period. Participants carried out the soccer-specific decision-making task developed

156 by Hüttermann, Smeeton et al. (2019) at three different exercise loads in a randomized order:

157 rest, moderate, and high - i.e. one third started with the rest condition, one third with the

158 moderate, and one third with the high load condition. Instructions were delivered on the screen,

159 and participants were given the opportunity to ask questions prior to starting the experiment.

160 Physical load. Previous research has reported that the mean duration of each 'purposeful'

161 movement in soccer lasts about $13 \mathrm{~s}$, while the mean time between 'purposeful' movements is

162 20s (all spent on a low intensity level). This finding demonstrated a mean ratio of 1:1.6. (Note

163 though that this ratio is not to be confused with the 'physiological work: rest' - ratio, because

164 some purposeful movements also included low intensity movements (Bloomfield, Polman, \&

165 O’Donoghue, 2007)). In accordance with previous research examining the effects of moderate- 
166 and high-intensity exercise on cognitive performance (e.g., Smith et al., 2016), we determined

167 the resting heart rate $\left(\mathrm{HR}_{\mathrm{rest}}\right)$ as well as the maximum heart rate $\left(\mathrm{HR}_{\max }\right)$ for each participant to

168 calculate $70 \%$ and $90 \%$ of the individual heart rate reserve (HRR) before the implementation of

169 the soccer task. HR rest was obtained while the participant was lying down in a supine position

170 and wearing the heart rate monitor in a quiet room for 3 minutes. For male participants the $\mathrm{HR}_{\max }$

171 was estimated as 220 minus their age; for female participants the HRmax was estimated as 226

172 minus their age (Beashel, Sibson, \& Taylor, 2001). Afterwards, we calculated HRR as the

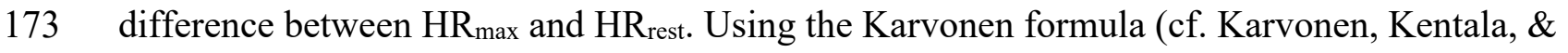

174 Mustala, 1957), we calculated the exercise heart rates at $70 \%$ and $90 \%$ target load (e.g.,

175 Exercise $\left.\mathrm{HR}=70\left(\mathrm{HR}_{\max }-\mathrm{HR}_{\text {rest }}\right)+\mathrm{HR}_{\text {rest }}\right)$. Previous research has shown that this calculation

176 gives an exercise intensity that is equivalent to the desired percentage of $\mathrm{VO}_{2} \mathrm{R}$ (maximal oxygen

177 uptake reserve, i.e. the difference between resting and maximal VO2; Swain \& Leutholtz, 1997).

178 The Borg's (1970) scale was used as an additional rating of perceived (subjective)

179 exertion (RPE) and was indicated by each participant after each of the three test conditions. It is

180 a scientifically validated method for estimating feelings of exertion. On this scale physical

181 exertion can be rated on a range that varies from 6 (no exertion at all) to 20 (maximal exertion).

182 Participants were asked to rate their perceived exertion based on the strain on and the fatigue of

183 their musculature as well as their breathlessness (or shortness of breath). In order to familiarise

184 the participants with the magnitude of the values on the RPE scale, they were given the

185 following verbal 'anchors' - number 9 corresponds with very light effort/exertion, for example,

186 normal walking at one's preferred pace - 13 on the scale indicates that the task is somewhat

187 exhausting, but one could continue the task at the current level of load rather easily - 15 is

188 strenuous and difficult, but one could still continue - 17 signifies that the level of physical load is

189 very exhausting and that continuation is still possible, but one would have to exert great effort 
190 and would be fatigued within a short time. Participants were asked to indicate their RPE as

191 honest as possible and without pondering.

192 The increasing HR (70\% and 90\% of individual HRR) were achieved by incrementally

193 increasing the resistance of the cycle ergometer until participants reached their individually

194 required target heart rates. Participants warmed up for a period of $5 \mathrm{~min}$. After the respective

195 target heart rates were reached, the soccer task started while participants continued their exercise.

196 The experimenter constantly ensured that participants stayed at their predetermined target heart

197 rates during the presentation of the trials $(+/-3 \mathrm{bpm})$. In between the trials participants were

198 allowed to reduce the level of physical load, and therefore their heart rate, for a few seconds

199 (while ensuring that they would reach their required target heart rate after a maximum of 20

200 seconds - based on the previously described mean ratio between physical load and rest duration

201 in soccer games, see Bradley et al., 2009; a watch with a second hand was visible for the

202 participants), before they had to return to their target heart rate to judge the next game situation.

203 Soccer-specific decision-making task. The task was presented using Delphi XE 3. Participants

204 completed the soccer-specific task under each of the three different physical load conditions

205 (rest, moderate load, high load) in randomized order. In each of the three conditions, participants

206 performed 24 trials, preceded by 2 additional practice trials. A central fixation cross (1000ms)

207 appeared at the beginning of each trial. Two stimuli were subsequently presented for $300 \mathrm{~ms}$

208 equidistant from and on opposite sides of the fixation cross (see Figure 2). Stimuli were

209 randomly presented at one of eight horizontal visual angles from the participant's view $\left(20^{\circ}, 40^{\circ}\right.$,

$21060^{\circ}, 80^{\circ}, 100^{\circ}, 120^{\circ}, 140^{\circ}, 160^{\circ}$ ) and were equally likely to appear at each visual angle. Each

211 stimulus consisted of different player configurations (the players' height was approximately

$21230 \mathrm{~cm}$ ) including one teammate being randomly surrounded by zero, one, two, or three opposing

213 players. The teammates' body postures indicated the direction they were moving to (either 
214 towards the centre of the pitch or towards the sideline, i.e. the centre or the outer end of the

215 screen, respectively). The opposing players always moved towards the respective teammate on

216 each of the participant's sides.

217 In each trial, participants were required to imagine they were the player in possession of

218 the ball and to decide whether it would be best to pass the ball to one of the teammates or to

219 stop/control the ball. The challenge was to only pass the ball to the left or right side if they

220 perceived a teammate who was running in their direction (towards the centre) and was not

221 surrounded by any opponent players. In contrast, participants should decide to not pass the ball

222 when a teammate was running towards the sideline and/or was surrounded by at least one

223 opponent player. Participants were asked to verbally report their decision (pass to the left, pass to

224 the right, no pass) as fast as possible, but at least within a time limit of $3 \mathrm{sec}$ (otherwise the trial

225 was considered a mistake). Afterwards, they had to specify the teammates' running directions for

226 each side and the number of opponent players surrounding each teammate. (Note: Each stimulus

227 had to be considered independently as the number of opponent players and the running direction

228 of teammates could differ for each side. In Figure 2, for example, teammates were surrounded by

229 two opponent players at both sides, but while the teammate at the left side was running towards

230 the center, the teammate at the right side was running towards the sideline.).

\section{Data analysis}

232 In total, we analysed main task performance (accuracy rate) by summing up the trials in

233 which all three subtasks were solved correctly (i.e. the decision-making task, the feature-

234 recognition task, and the object-detection task; performance was also calculated for each of the

235 three subtasks separately). For the main task and for each subtask we conducted an $8 \times 3$

236 repeated measures analysis of variance (ANOVA) with visual angle $\left(20^{\circ}, 40^{\circ}, 60^{\circ}, 80^{\circ}, 100^{\circ}\right.$,

$237120^{\circ}, 140^{\circ}$, or $160^{\circ}$ ) and exercise load (rest, $70 \%$ of individual HRR, $90 \%$ of individual HRR) as 
238 the within-participant factors and accuracy rate (performance) in the respective task as dependent

239 variable. When Mauchly's test revealed violations of the sphericity assumption for any of the

240 variables, we used adjusted degrees of freedom based on the Greenhouse-Geisser correction. In

241 the feature-recognition and object-detection task, responses were only treated as correct when

242 participants reported the correct answer for stimuli at both sides (left, right).

\section{Results}

244 Physical load. Overall, we measured a mean resting heart rate ( $\left.\mathrm{HR}_{\mathrm{rest}}\right)$ of $64.37 \mathrm{bpm}(S D=4.27$

$245 \mathrm{bpm})$ and calculated a mean maximum heart rate $\left(\mathrm{HR}_{\max }\right)$ of $197.30 \mathrm{bpm}(S D=2.28 \mathrm{bpm})$. Using

246 the Karvonen formula, we calculated a mean exercise heart rate of $157.42 \mathrm{bpm}(S D=2.30 \mathrm{bpm})$

247 at $70 \%$ target $\mathrm{HR}$ and of $184.01 \mathrm{bpm}(S D=2.20 \mathrm{bpm})$ at $90 \%$ target intensity. The subjective

248 Borg rating was significantly different between the high $(M=18.17, S D=0.75)$ and moderate

$249(M=13.87, S D=0.90)$ load levels, $t(29)=23.842, p<.001, d=4.353$.

250 Total score. The total percentage of correct responses, in which all three tasks (decision-making

251 task; feature-recognition task; object-detection task) were answered correctly, averaged across all

252 three exercise conditions, was $43.33 \%(S D=7.26 \% \text {; see Figure } 3)^{1}$. The ANOVA revealed a

253 main effect of visual angle, $F(4.307,124.902)=36.011, p<.001, \eta^{2}=.554, \varepsilon=.615$ (Mauchly's

254 test of sphericity: $\left.\chi^{2}(27)=64.293, p<.001\right)$, demonstrating that accuracy decreased with

255 increasing visual angles. There was a significant effect of exercise load (rest: $M=44.86 \%, S D=$

$25614.25 \%$; $70 \%$ HRR: $M=47.08 \%, S D=13.14 \%$; $90 \%$ HRR: $M=38.06 \%, S D=9.95 \%$ ),

$257 F(2,58)=4.193, p=.020, \eta^{2}=.126, \varepsilon=.631$; we performed follow-up comparisons (Bonferroni

258 corrected adjusted alpha of 0.017 ) indicating that participants performed better under moderate

259 exercise load, compared to the high load condition, $t(29)=2.863, p=.008, d=.523$, with no

260 difference between moderate load and rest condition, $t(29)=0.729, p=.472$, nor between the

261 rest and high load condition, $t(29)=1.930, p=.063$. Furthermore, the ANOVA did not reveal a 
262 significant interaction between visual angle and exercise load, $F(8.835,256.201)=0.980, p=$ 263.456 (Mauchly's test of sphericity: $\chi^{2}(104)=135.677, p=.029$ ).

264 Decision-making subtask. In total, participants made the correct decision (pass to the left, no 265 pass, pass to the right) in $91.16 \%(S D=4.47 \%)$ of the trials averaged across all exercise loads. 266 Decision-making performance decreased with increasing visual angles between the stimuli (or 267 more specifically remained high until the visual angle was increased beyond a certain point), $268 F(3.282,95.174)=34.748, p<.001, \eta^{2}=.545, \varepsilon=.469$ (Mauchly's test of sphericity: $\chi^{2}(27)=$ $269130.849, p<.001)$. Participants performed comparably well under all three exercise loads, $270 F(2,58)=1.962, p=.150$. We did not find a significant interaction between angle and exercise 271 load, $F(6.020,174.566)=1.586, p=.154, \varepsilon=.430$ (Mauchly's test of sphericity: $\chi^{2}(104)=$ $272 \quad 259.109, p<.001)$.

273 Feature-recognition subtask. A correct response in a trial in the feature-recognition task 274 required accurate reporting of the running direction of the teammates at both sides of the 275 participant's visual field. In total, participants correctly identified the running direction of both 276 teammates in $52.36 \%(S D=6.10 \%)$ of all trials. The ANOVA revealed a significant main effect 277 for visual angle $F(4.532,131.439)=19.715, p<.001, \eta^{2}=.405, \varepsilon=.647$ (Mauchly's test of 278 sphericity: $\chi^{2}(27)=47.985, p=.008$ ), again indicating a decline of performance with increasing 279 angles. In addition, participants differed in accuracy across the three exercise loads, $F(2,58)=$ $2804.352, p=.017, \eta^{2}=.130$ (see Figure 4). In the feature-recognition task, participants attained 281 highest success rates at moderate load $(M=55.97 \%, S D=12.22 \%)$ compared to the high load 282 condition $(M=47.36 \%, S D=9.57 \%), t(29)=2.849, p=.008, d=.520$, with no difference 283 between moderate and rest condition $(M=53.75 \%, S D=12.10 \%), t(29)=0.731, p=.471$, nor 284 between rest and high load condition, $t(29)=2.110, p=.044$ (Bonferroni corrected adjusted 285 alpha of 0.017). Furthermore, the ANOVA did not reveal an interaction effect for visual angle 
286 and exercise load, $F(8.785,254.756)=0.623, p=.773, \varepsilon=.627$ (Mauchly's test of sphericity:

$\left.287 \quad \chi^{2}(104)=132.743, p=.042\right)$.

288 Object-detection subtask. A response in a trial in the object-detection task was considered 289 correct only if participants reported the accurate number of opponent players for both sides. In 290 total, participants attained an accuracy rate of $78.29 \%(S D=11.68 \%)$ across all trials. There 291 was a main effect for visual angle, $F(3.613,104.767)=44.893, p<.001, \eta^{2}=.608, \varepsilon=.516$ 292 (Mauchly's test of sphericity: $\chi^{2}(27)=67.992, p<.001$ ), pointing out that participants' accuracy 293 decreased from the centre to the periphery. Moreover, the ANOVA revealed a main effect of

294 exercise load, $F(2,58)=12.622, p<.001, \eta^{2}=.303$ (see Figure 5). Participants performed worse 295 under high load $(M=69.17 \%, S D=8.93 \%)$, compared to moderate load $(M=83.89 \%, S D=$ $29617.39 \%), t(29)=-4.337, p<.001, d=.792$, and to the rest condition $(M=81.81 \%, S D=$ $29718.13 \%), t(29)=-3.518, p=.001, d=.642$, with no difference between moderate load and rest 298 condition, $t(29)=0.869, p=.392$ (Bonferroni corrected adjusted alpha of 0.017$)$. We found a 299 significant interaction between exercise load and visual angle, $F(7.593,220.185)=11.046, p<$ $300.001, \eta^{2}=.276, \varepsilon=.753$ (Mauchly's test of sphericity: $\chi^{2}(104)=195.736, p<.001$ ). The decline 301 in performance, as a result of increasing visual angles, became more pronounced in the high load 302 compared to the rest or moderate load conditions (see Figure 3).

\section{Discussion}

This study investigated the impact of physical load on soccer players' perceptual and

305 attentional capabilities as well as on their decision making in soccer game situations. In support

306 of our predictions, the total performance in the soccer-specific task (i.e., the conjunction of the

307 feature-recognition, object-detection, and decision-making tasks) confirms previous study

308 results, in that performance in complex tasks can be influenced through different physical loads

309 placed upon athletes (e.g., McMorris et al., 1999; Royal et al., 2006; Tenenbaum et al., 1993). 
310 Whilst no differences in physical load condition were observed in the decision-making task,

311 there was a decline in feature-recognition performance at the high exercise load compared to the

312 moderate load condition and a decline in object-detection performance was found at the high

313 exercise load condition only when stimuli presentation exceeded 120 degrees of visual angle. It

314 is clear that when physiological arousal approaches a maximal level though performance

315 declines.

316 Apart from the total performance in the soccer-specific task, we analysed participants'

317 performances in the separate subtests (decision-making, feature-recognition, object-detection), in

318 order to understand how different dimensions of decision-making performance were influenced

319 by the physical load conditions. The results suggest that athletes' visual attention and perception

320 capabilities were affected by changes in physical exercise load. Overall, performances was

321 higher in the moderate ( $70 \%$ of HRR) compared to the high load condition ( $90 \%$ of HRR) but

322 not compared to the rest condition. This pattern of findings was consistent for attentional and

323 perceptual capabilities. However, the moderate load condition did not result in higher levels of

324 performance than the rest condition resulting in a performance-load curve that does not strictly

325 comply with an inverted-U shape.

326 But although athletes' attentional and perceptual capabilities were affected —at least

327 partially - by physical exercise loads, we did not find an impact on their sport-specific decision-

328 making performance. On the one hand, this result supports the findings of previous studies that

329 physical load does not impact the quality of the final decision (e.g., Hepler, 2015; Paradis et al.,

330 2016). It appears that while it is not possible to perceive all information in the peripheral field in

331 detail (e.g., the positioning or running direction of players), decision making is not negatively

332 affected (cf. Olde Rikkert et al., 2015) at least those decisions that have been required in the

333 decision-making task. However, on the other hand, there are also contradictory findings 
334 reporting a positive effect of physical exercise on decision making (e.g., Royal et al., 2006;

335 Tenenbaum et al., 1993). For example, Tenenbaum and colleagues (1993) observed improved

336 accuracy of decision making in handball players during aerobic exercise (11-12 METS). It is

337 difficult to draw clear conclusions as to why there are contradictory findings because the exercise

338 protocols do not compare exactly across studies. However, findings may be attributed to

339 different psychological tasks used in the different studies and experiences in making decisions

340 during exercise. Tenenbaum et al. (1993) used generic tests of short-term memory general

341 intelligence, attentional style, and concentration. In the present study, sport-specific stimuli were

342 used and the decision-making task to choose the correct opponent to pass to, matched the

343 experience of the participants. In fact, sport specific experience improved psychological task

344 performance in the Tenenbaum et al. (1993) study. The answer to the question of why

345 improvements in performance were seen in Tenenbaum et al. (1993) and not here could be

346 attributed to order effects, which were not controlled for in the exercise condition in Tenenbaum

347 et al. (1993). Therefore, there may have been a warm-up effect on the psychological tasks

348 confounded performance in the exercise conditions. In this study, exercise load was

349 counterbalanced and tasks differed. However, it should be considered that attentional processes

350 in real soccer game situations are oftentimes more complex than the challenge to decide whether

351 to pass the ball to the left, to the right, or whether to control it/not pass at all. Considering these

352 and further factors, future studies should investigate the influence of physical load on the

353 decisional behaviour of athletes as a function of task specificity and complexity.

354 Differences in performance were found between the high and moderate load conditions in

355 the feature-recognition and object-detection task. The high load condition was chosen to extend

356 previous research by Hüttermann and Memmert (2014), who showed an inverted-U relationship

357 between the intensity of physical load and cognitive performance for non-athletes, and a linear 
358 relation for athletes. However, intensities were 50, 60, and $70 \%$ of the age-dependent predicted

359 maximal heart rate. The linear relationship between exercise intensity and visual attention was

360 explained by the fact that an intensity level of $70 \%$ was rather a moderate than a high intensity

361 for trained athletes; and it was assumed that higher intensities may lead to a decline in

362 performance. Participants in the current study performed the decision-making task with $70 \%$ and

$36390 \%$ of heart rate reserve, as well as under rest. It was found that perceptual and attentional

364 performance declined at the highest load. As participants reported RPE values of 14 for $70 \%$

365 exercise HRR and 18 for $90 \%$ exercise HRR on the Borg scale ranging from 6 (no exertion at

366 all) to 20 (maximal exertion), we can assume that the targeted intensity levels were reached. It

367 remains a task for future studies to test to what degree the performance in the soccer-specific task

368 can be influenced by different intensities of physical load as a function of the performance- and

369 fitness-levels of participating athletes. Further, the physical load ratios could be adapted

370 according to the divisions the athletes are active in.

371 An interaction between visual angle and physical load was found in the object-detection

372 subtask but not in the other subtasks. Performance in this task declined more rapidly in the 120

373 degree visual angle condition in the $90 \%$ physical load condition than in the $70 \%$ condition and

374 more rapidly in both $70 \%$ and rest conditions for visual angles greater than 120 degrees. This

375 result was not expected, and any attempted explanation, therefore, has to be considered as a post-

376 hoc rationalisation. This finding supports the assumption that the object-detection subtask has

377 elements that are independent of the other two tasks because this interaction was not found in the

378 decision-making or the feature-recognition subtask. However, the authors are not aware of any

379 literature to help explain why exercise at a high load differentially affected the object-detection

380 subtask, but not the feature-recognition or decision-making subtasks. Potentially, this interaction

381 effect in the subtask might also be explained by increases in statistical power. Alternatively, in 
382 contrast to decision-making or attentional processes, perceptual processes in the extremities of

383 the peripheral vision may be differently affected by exercising at high intensity than in other

384 parts of the visual field. However, systematic, hypothesis-driven, future research is needed to

385 provide an evidence-based explanation of this finding.

386 The current study does not come without limitations. To simulate the changing load

387 intensities found in soccer match-play (cf. Bloomfield et al., 2007), participants were required to

388 maintain their target heart rate from onset of the presentation of each trial until their

389 ratings/responses for the respective game situation. Subsequently, they were given a few seconds

390 to reduce the load at their own discretion (however, they had to continue pedalling), before

391 returning to their target heart rate and being presented with the next game situation. Participants

392 used the time between the trials in different ways, meaning some participants reduced the load

393 intensity more than others did. Even though load levels also vary among different players in real

394 soccer games, future studies should try to achieve a better comparability of results by

395 predetermining a standardized load level for the breaks between the trials. Nevertheless, it should

396 be noted here that a cessation of exercise of a few seconds (after the 20 seconds participants had

397 to reach the required target heart rate), in between the predetermined loads, is a relatively short

398 time period, during which no great changes in the participants' heart rates were seen compared to

399 the predetermined target heart rate.

400 In the current study, decision making was analysed as the accuracy of decisions, i.e., we

401 measured the quality of decisions, but not the speed of decision making. As previous studies

402 have found no impact of physical arousal on the quality but on the speed of decision making

403 (e.g., Hepler, 2015), the integration of a reaction time measure might be a potential avenue for

404 future research. Another potential avenue of investigation could involve further manipulations of 405 the task demands, such as the integration of dynamic game scenes (e.g., moving and looming 
406 stimuli) instead of static pictures. In the current study, participants performed the decision-

407 making task while bicycling on an ergometer, although treadmill running would better

408 correspond to the natural demands on soccer players. (In addition, it took some time until every

409 participant was able to finally reach the $90 \%$ exercise HRR using the bicycle ergometer.) Future

410 research should search for alternatives ensuring both the safety of the participants (this may

411 indeed be a problem using a treadmill) and the possibility of exercising at high intensities.

412 Although decision-making performance did not decrease with high exercise load, it

413 would be interesting to train the players' cognitive skills under physical load in future research in

414 order to investigate whether this training would have a positive effect on the players' decision

415 making in soccer (see Alder, Broadbent, Stead, \& Poolton, 2019, for a study in badminton).

416 There is research demonstrating training approaches for visual attentional capabilities (e.g.,

417 Hüttermann \& Memmert, 2018), perceptual attentional capabilities (e.g., Swart et al., 2012), and

418 decision making (e.g., Hepler, 2015) in sport athletes, however, future research might develop

419 specific programs integrating all of these cognitive skills/tasks in one training.

420 In summary, the findings of the current study suggest that different physical exercise

421 loads can temporarily affect attentional and perceptual capabilities of sport athletes, but they do

422 not positively or negatively affect their sport-specific decision making. Depending on the

423 complexity of the decision-making process, in future, training possibilities should not only be

424 considered for attentional and perceptual skills, but also, for sport athletes' decision-making

425 skills, in order to train the skills needed to meet the cognitive demands on sportspeople as

426 comprehensively as possible. 
429 Aks, D. J. (1998). Influence of exercise on visual search: Implications for mediating cognitive mechanisms. Perceptual and Motor Skills, 87, 771-783.

431 Alder, D. B., Broadbent, D. P., Stead, J., \& Poolton, J. (2019). The impact of physiological load on anticipation skills in badminton: From testing to training. Journal of Sports Sciences, 37, 1816-1823.

Allard, F., Brawley, L., Deakin, J., \& Elliot, F. (1989). The effect of exercise on visual attention performance. Human Performance, 2, 131-145.

Arcelin, R., Brisswalter, J., \& Delignières, D. (1997). Effect of physical exercise duration on decisional performance. Journal of Human Movement Studies, 32, 123-140.

Beashel, P., Sibson, A., \& Taylor, J. (2001). The world of sport examined (2nd ed). Thomas Nelson and Sons Ltd: UK.

Bloomfield, J., Polman, R., \& O’Donoghue, P. (2007). Physical demands of different positions in FA premier league soccer. Journal of Sports Science and Medicine, 6, 63-70.

Borg, G. (1970). Perceived exertion as an indicator of somatic stress. Scandinavian Journal of

444 Bradley, P. S., Sheldon, W., Wooster, B., Olsen, P., Boanas, P., \& Krustrup, P. (2009). Highintensity running in English FA Premier League soccer matches. Journal of Sports Sciences, 27, 159-168.

447 Chmura, J., Nazar, K., \& Kaciuba-Uscilko, H. (1994). Choice reaction time during graded exercise in relation to blood lactate and plasma catecholamine thresholds. International

450 Dalen, T., Ingebrigtsen, J., Ettema, G., Havard, G. H., \& Wisløff, W. (2016). Player load, acceleration, and deceleration during 45 competitive matches of elite soccer. Journal of 
Strength and Conditioning Research, 30, 351-359.

453 Delignières, D., Brisswalter, J., \& Legros, P. (1994). Influence of physical exercise on choice

Faul, F., Erdfelder, E., Buchner, A., \& Lang, A.-G. (2009). Statistical power analyses using reaction time in sports experts: The mediating role of resource allocation. Journal of Human Movement Studies, 27, 173-188. G*Power 3.1: Tests for correlation and regression analyses. Behavior Research Methods, 41, 1149-1160.

Hepler, T. J. (2015). Decision-making in sport under mental and physical stress. International Journal of Kinesiology and Sports Science, 3, 79-83.

Humphreys, M. S., \& Revelle, W. (1984). Personality, motivation, and performance: A theory of the relationship between individual differences and information processing. Psychological Review, 91, 153-184.

Hüttermann, S., \& Memmert, D. (2014). Does the inverted-U function disappear in expert athletes? An analysis of the attentional behavior under physical exercise of athletes and non-athletes. Physiology and Behavior, 131, 87-92.

Hüttermann, S., \& Memmert, D. (2017). The attention window: A narrative review of limitations and opportunities influencing the focus of attention. Research Quarterly for Exercise and Sport, 88, 169-183.

Hüttermann, S., \& Memmert, D. (2018). Effects of lab- and field-based attentional training on athletes' attention-window. Psychology of Sport and Exercise, 38, 17-27.

Hüttermann, S., Ford, P. R., Williams, A. M., Varga, M., \& Smeeton, N. J. (2019). Attention, perception, and action in a simulated decision-making task. Journal of Sport and Exercise Psychology, 41, 230-241.

Hüttermann, S., Memmert, D., \& Simons, D. J. (2014). The size and shape of the attentional 
476

477

478

479

480

481

482

483

484

485

486

487

488

489

490

491

492

493

494

495

496

497

498

499

"spotlight" varies with differences in sports expertise. Journal of Experimental Psychology: Applied, 20, 147-157.

Hüttermann, S., Memmert, D., Simons, D. J., \& Bock, O. (2013). Fixation strategy influences the ability to focus attention on two spatially separate objects. PLoS ONE, 8, e65673.

Hüttermann, S., Smeeton, N. J., Ford, P. R., \& Williams, A. M. (2019). Colour perception and attentional load in dynamic, time-constrained environments. Frontiers in Psychology, 9, 2614.

Karvonen, M. J., Kentala, E., \& Mustala, O. (1957). The effects of training heart rate: A Longitudinal Study. Annales Medicinae Experimentalis et Biologiae Fenniae, 35, 307315.

Klatt, S., \& Smeeton, N. J. (2019). Immersive screens change attention width but not perception or decision-making performance in natural and basic tasks. Applied Ergonomics, 82, 102961.

Klatt, S., \& Smeeton, N. J. (2020). Visual and auditory information during decision making in sport. Journal of Sport and Exercise Psychology, 42, 15-25.

Labelle, V., Bosquet, L., Mekary, S., \& Bherer, L. (2013). Decline in executive control during acute bouts of exercise as a function of exercise intensity and fitness level. Brain and Cognition, 81, 10-17.

Lambourne, K., \& Tomporowski, P. D. (2010). The effect of exercise-induced arousal on cognitive task performance: A meta-regression analysis. Brain Research, 1341, 12-24.

Lambourne, K., Audiffren, M., \& Tomporowski, P. D. (2010). Effects of acute exercise on sensory and executive processing tasks. Medicine and Science in Sports and Exercise, 42, 1396-1402.

McMorris, T., \& Graydon, J. (1996). The effect of exercise on the decision-making performance 
of experienced and inexperienced soccer players. Research Quarterly for Exercise and Sport, 67, 109-114.

McMorris, T., \& Graydon, J. (1997). The effect of exercise on cognitive performance in soccerspecific tests. Journal of Sports Sciences, 15, 459-468.

McMorris, T., \& Graydon, J. (2000). The effect of incremental exercise on cognitive performance. International Journal of Sport Psychology, 31, 66-81.

McMorris, T., \& Keen, P. (1994). Effect of exercise on simple reaction times of recreational athletes. Perceptual and Motor Skills, 78, 123-130.

McMorris, T., Myers, S., MacGillivary, W. W., Sexsmith, J. R., Fallowfield, J., Graydon, J., \& Forster, D. (1999). Exercise, plasma catecholamine concentrations and decision-making performance of soccer players on a soccer-specific test. Journal of Sports Sciences, 17,

McMorris, T., Sproule, J., Turner, A., \& Hale, B. J. (2011). Acute, intermediate intensity exercise, and speed and accuracy in working memory tasks: A meta-analytical comparison of effects. Physiology and Behavior, 102, 421-428.

Olde Rikkert, J. O., De Haes, V., Barsingerhorn, A. D. Theelen, T., \& Olde Rikkert, M. G. M. (2015). The colour of a football outfit affects visibility and team success. Journal of Sports Sciences, 33, 2166-2172.

Osgnach, C., Poser, S., Bernardini, R., Rinaldo, R., \& Di Prampero, P. E. (2010). Energy cost and metabolic power in elite soccer: A new match analysis approach. Medicine and Science in Sports and Exercise, 42, 170-178.

Paradis, K., Larkin, P., \& O’Connor, D. (2016). The effects of physical exertion on decisionmaking performance of Australian football umpires. Journal of Sports Sciences, 34, 1535-1541. 
524 Pesce, C., Cereatti, L., Casella, R., Baldari, C., \& Capranica, L. (2007). Preservation of visual

525

526

527

528

529

530

531

532

533

534

535

536

537

538

539

540

541

542

543

544

545

546

547 attention in older expert orienteers at rest and under physical effort. Journal of Sport and Exercise Psychology, 29, 78-99.

Royal, K. A., Farrow, D., Mujika, I., Halson, S. L., Pyne, D., \& Abernethy, B. (2006). The effects of fatigue on decision making and shooting skill performance in water polo players. Journal of Sports Sciences, 24, 807-815.

Russell, M., Sparkes, W., Northeast, J., Cook, C. J., Love, T. D., Bracken, R. M., \& Kilduff, L. P. (2014). Changes in acceleration and deceleration capacity throughout professional soccer match-play. Journal of Strength and Conditioning Research, 30, 2839-2844.

Sanders, A. F. (1983). Towards a model of stress and human performance. Acta Psychologica, $53,61-97$.

Sarmento, H., Marcelino, R., Anguera, M. T., CampaniCo, .J, Matos, N., \& LeitAo, J. C. (2014). Match analysis in football: A systematic review. Journal of Sports Sciences, 32, 18311843.

Smith, M., Tallis, J., Miller, A., Clarke, N. D., Guimaraes-Ferreira, L., \& Duncan, M. J. (2016). The effect of exercise intensity on cognitive performance during short duration treadmill running. Journal of Human Kinetics, 51, 27-35.

Swain, D. P., \& Leutholtz, B. C. (1997). Heart rate reserve is equivalent to $\% \mathrm{VO}_{2}$ reserve, not to \%VO2max. Medicine and Science in Sports and Exercise, 29, 410-414.

Swart, J., Lindsay, T. R., Lambert, M. I., Brown, J. C., \& Noakes, T. D. (2012). Perceptual cues in the regulation of exercise performance - physical sensations of exercise and awareness of effort interact as separate cues. British Journal of Sports Medicine, 46, 42-48.

Tenenbaum, G., Yuval, R., Elbaz, G., Gar-Eli, M., \& Weinberg, R. (1993). The relationship between cognitive characteristics and decision making. Canadian Journal of Applied 
Physiology, 18, 48-62.

549 Tomporowski, P. D. (2003). Effects of acute bouts of exercise on cognition. Acta Psychologica, 550 $112,297-324$.

551 Yerkes, R. M., \& Dodson, J. D. (1908). The relation of strength of stimulus to rapidity of habitformation. Journal of Comparative Neurology and Psychology, 18, 459-482.

553 Zurutuza, U., Castellano, J., Echeazarra, I., \& Casamichana, D. (2017). Absolute and relative 554 training load and its relation to fatigue in football. Frontiers in Psychology, 6, 878. 
$557{ }^{1}$ Although this accuracy rate seems to be very low at first sight, it should be considered that a 558 trial was only evaluated as correct when participants made the correct decision and gave correct 559 answers in the feature-recognition and object-detection task — also including situations with 560 visual angles of up to $160^{\circ}$, i.e. lying outside the maximal shift of attention measured in previous 561 research (e.g., Hüttermann, Memmert, \& Simons, 2014; Hüttermann, Memmert, Simons, \& 562 Bock, 2013). 
Figure legends

565 Figure 1. The figure shows the experimental setup with a subject sitting on the bicycle ergometer 566 in front of the $2.4 \times 6.0 \mathrm{~m}$ IGLOO dome.

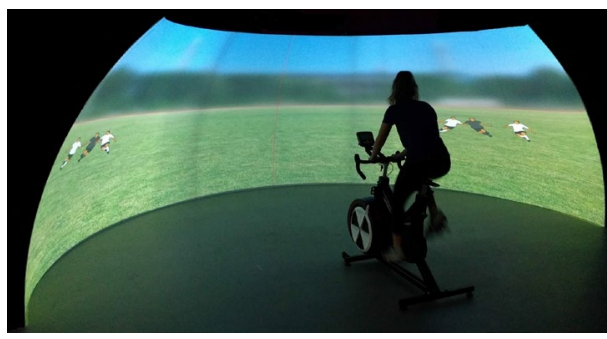

568 Figure 2. Sequence of events in one exemplary trial.

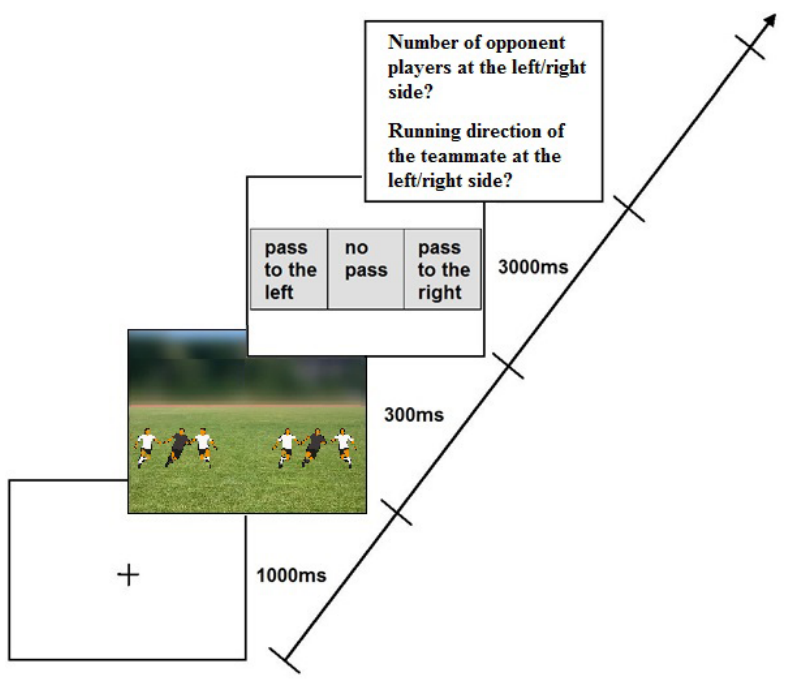

570 Figure 3. Percentage of participants' total accuracy rates, their decision making, the

571 identification rates of the teammates' running direction, and the identification rates of the

572 number of opponents in the soccer decision-making task, in degrees of visual angle as a function

573 of physical exercise intensity (rest, moderate, high). Symbols represent across-participant means,

574 and error bars show standard deviations. (Note: ${ }^{*} p<.017 ; * *<<.001$; Bonferroni corrected post-

575 hoc comparisons had an adjusted alpha of 0.017 . Y-axis scale adjusted to $120 \%$ to allow plotting 576 of error bars) 

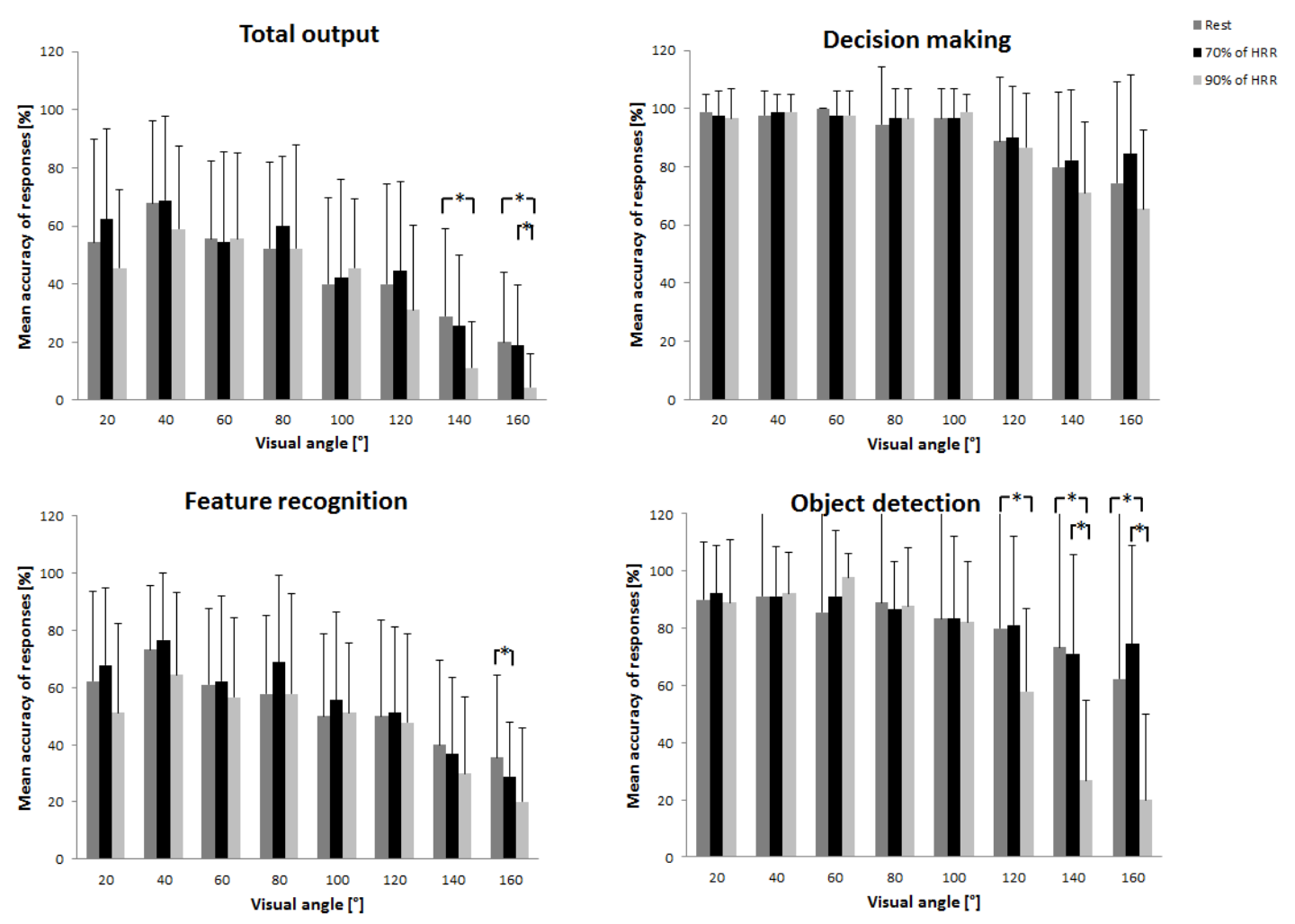

Figure 4. Percentage of participants' accuracy rates in the feature-recognition subtask

579 (identification rate of the teammates' running direction) as a function of physical exercise

580 intensity (rest, moderate, high). Symbols represent across-participant means, and error bars show

581 standard deviations. (Note: ${ }^{*} p<.017$; Bonferroni corrected post-hoc comparisons had an adjusted 582 alpha of 0.017.$)$

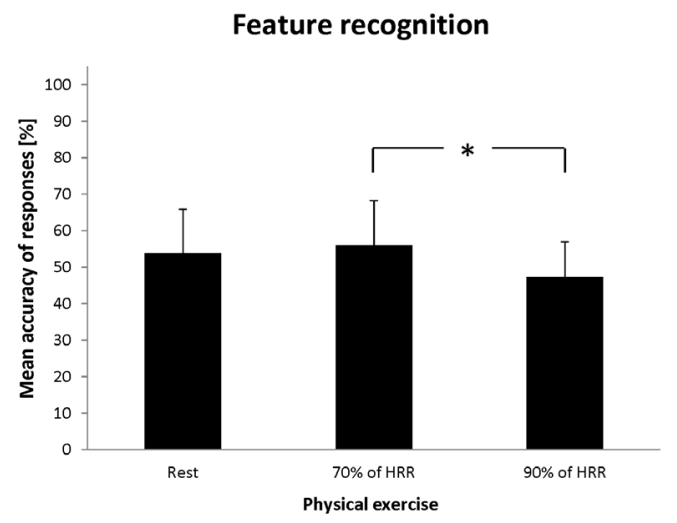

584 Figure 5. Percentage of participants' accuracy rates in the object-detection subtask (identification 
585 rate of the number of opponents) as a function of physical exercise intensity (rest, moderate,

586 high). Symbols represent across-participant means, and error bars show standard deviations.

587 (Note: ${ }^{*} p<.017$; Bonferroni corrected post-hoc comparisons had an adjusted alpha of 0.017.)

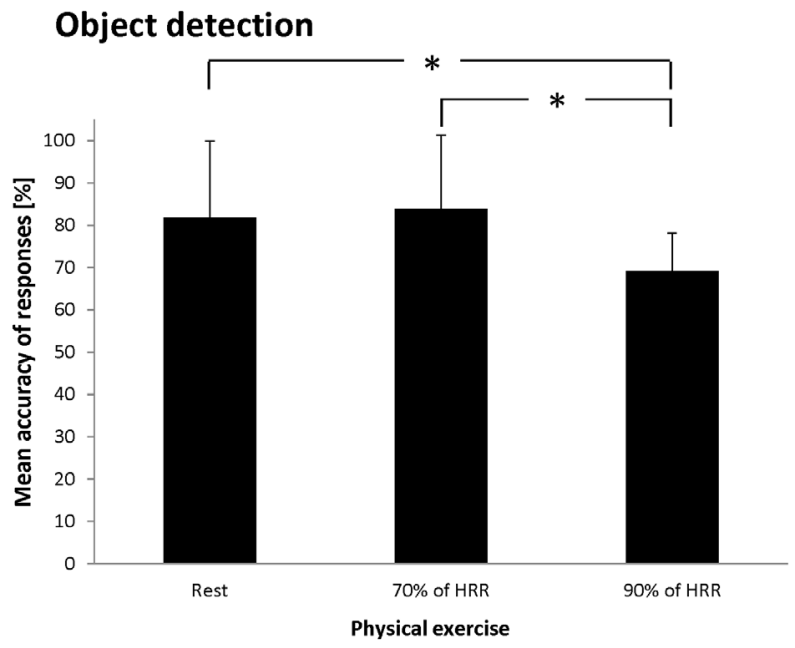

